\title{
Avaliação da ecoeficiencia na intermodalidade para o transporte regional de carga - o caso do calcário siderúrgico
}

\author{
Cintia Machado de Oliveira $^{1}$, Marcio de Almeida D'Agosto², \\ Guilherme Anselmo Guimarães dos Santos ${ }^{3}$, George Vasconcelos Goes ${ }^{4}$, \\ Lino Guimarães Marujo ${ }^{5}$, Daniel Neves Schmitz Gonçalves ${ }^{6}$, Mariane Gonzalez da Costa ${ }^{7}$
}

${ }^{1}$ Centro Federal de Educação Tecnológica Celso Suckow da Fonseca, cintia.machado.oliveira.1@gmail.com

${ }^{2}$ Programa de Engenharia de Transportes (PET/Coppe), UFRJ, dagosto@pet.coppe.ufrj.br

3Programa de Engenharia de Transportes (PET/Coppe), UFRJ, guianselmo@yahoo.com.br

4Programa de Engenharia de Transportes (PET/Coppe), UFRJ, george.vasconcelos@outlook.com

${ }^{5}$ Programa de Engenharia de Produção (PEP/Coppe), UFRJ, Igmarujo@poli.ufrj.br

6Programa de Engenharia de Transportes (PET/Coppe), UFRJ, daniel.schmitz.jf@gmail.com

7Programa de Engenharia de Transportes (PET/Coppe), UFRJ, marigonzalez@poli.ufrj.br

\section{Recebido:}

17 de abril de 2019

Aceito para publicação:

10 de outubro de 2019

Publicado:

XX de novembro de 2019

Editor de área:

Renato Lima

\section{Palavras-chaves:}

Escolha modal,

Avaliação de desempenho,

Produto de baixo valor agregado,

Transporte e Logística.

\section{Keywords:}

Modal choice,

Performance evaluation,

Low product added value,

Transportation and Logistics.

DOI:10.14295/transportes.v27i3.2016

\begin{abstract}
RESUMO
Objetivou-se propor um procedimento que possibilite a escolha de alternativas para o transporte de produto de baixo valor agregado que apresente desempenho superior à alternativa de transporte especializado. Tal procedimento mostrou-se simples, dependendo apenas da disponibilidade de dados que podem ser obtidos a partir do monitoramento das operações de transporte e da estimativa de fatores de emissão de poluentes atmosféricos e GEE que podem ser obtidos em inventários de emissões produzidos por instituições públicas. A aplicação do procedimento ao caso do transporte de calcário, na Região Sudeste do Brasil, demonstrou que a alternativa de transporte especializado, embora atenda aos requisitos tradicionais do transporte de produtos de baixo valor agregado, por utilizar o transporte ferroviário por cerca de $90 \%$ do percurso, apresenta desempenho inferior a duas das alternativas não usuais de transporte. Ao introduzir o aspecto ambiental verificou-se que a melhor escolha seria a alternativa de transporte não usual do tipo rodo-ferro-rodo com container.
\end{abstract}

\section{ABSTRACT}

The aim to propose a procedure that allows a choice of alternatives for transporting low value-added product which provides higher performance than the alternative of specialized transport. This procedure proved to be simple, depending on the availability of the data obtained from the monitoring of transport operations and estimation of GHG and air pollutants emission that can be obtained in emission inventories produced by public institutions. The application of the procedure in the case of transport of limestone, in the southeast of Brazil, showed that the specialized transport alternative, although meets the traditional requirements of low value-added products transportation by using rail transport for about $90 \%$ of the route, presents worse performance than two unusual transport alternatives. By introducing the environmental aspect in the performance evaluation of transport alternatives it was found that the best choice would be unusual transport alternative, being the only that maintains better performance than unusual container transport alternative.

\section{INTRODUCÃO}

0 consumo energético final do Brasil tem no setor de transportes aproximadamente $34 \%$ da 
sua representatividade (EPE, 2017). Dessa participação, estima-se que o modo rodoviário (passageiro e carga) consuma 93,69\% da energia final, enquanto o modo ferroviário consome 1,37\% da energia utilizada pelo setor de transportes (Pereira Jr, 2018). Além disso, a intensidade energética do modo ferroviário de carga é aproximadamente de $123 \mathrm{~kJ} / \mathrm{t} . \mathrm{km}$, enquanto o modo rodoviário consome uma média próxima de $1.739 \mathrm{~kJ} / \mathrm{t} . \mathrm{km}$ (Gonçalves e D’Agosto, 2017). Apesar disso, no Brasil, a malha ferroviária é de somente $28.000 \mathrm{~km}$ (ANTF, 2014), destes, somente $9.800 \mathrm{~km}$ da malha são efetivamente utilizados (majoritariamente pelas companhias EFC, MRS e EFVM), enquanto 7.800 km são subutilizados e $10.400 \mathrm{~km}$ da malha não são utilizados.

Apesar da eficiência no uso da energia pelo modo ferroviário, a matriz de transporte brasileira é concentrada no modo rodoviário, impactando o desempenho do transporte multimodal de carga. Convencionalmente, os serviços ferroviários e por embarcação (aquático) são oferecidos no Brasil apenas na existência de um volume de carga suficiente e um negócio potencialmente lucrativo para o operador de transporte (ANTT, 2016). Convencionalmente, esse raciocínio tem sido aplicado no transporte do setor primário (commodities) na existência de um ramal ferroviário que conecte os pontos de origem e destino. No entanto, em países emergentes, o conceito de sincromodalidade, que é a integração inteligente dos modos de transporte. (Marcucci, 2014), não tem sido aplicado, tanto pelo poder público como pela iniciativa privada.

No contexto do embarcador, a definição sobre a estratégia de suprimento mais atrativa nos aspectos econômico e ambiental (ecoeficiência) depende, sobretudo, da infraestrutura de transporte disponível e da eficiência das plataformas intermodais. Esta situação exige que o embarcador disponha de um procedimento que permita a avaliação destas alternativas de forma a garantir custos e níveis de serviço semelhantes ao que se obteria por meio do transporte convencional para commodities (ferrovia), sem comprometer o desempenho de aspectos ambientais associados à: (i) intensidade energética; e (ii) emissão de poluentes atmosféricos e de gases de efeito estufa (GEE).

Na última década, o conhecimento sobre a redução das emissões de GEE do transporte rodoviário tem crescido substancialmente, desta forma, tem-se repensado a forma de se transportar carga pelo modo rodoviário através do conceito de ecoeficiência (Bektas et al., 2018; Guajardo et al., 2018).

Este artigo busca avaliar alternativas para o transporte de produtos primários (de baixo valor agregado), oferecendo ganhos em ecoeficiência por meio do desenvolvimento de um protocolo de avaliação. Para isso, conduziu-se uma revisão bibliográfica sobre os métodos, a estrutura e as variáveis empregadas para a avaliação de desempenho dos modos de transporte especializados e alternativas de transporte não usuais para o transporte de produtos de baixo valor agregado. Após isso, o protocolo desenvolvido é aplicado para avaliar as estratégias de suprimento de calcário siderúrgico na Região Sudeste do Brasil, ou seja, em um cenário de economia emergente.

\section{REVISÃO DA LITERATURA}

0 processo siderúrgico é considerado grande consumidor de energia de origem fóssil e consequentemente um grande emissor de poluentes atmosféricos locais e GEE (Weigel et al, 2016). Uma das fontes consumidoras dessa energia é o transporte dos insumos, que utiliza predominantemente óleo diesel de petróleo (Wadud, 2016). Nessa linha, a avaliação de desempenho é um procedimento para a escolha da melhor combinação de alternativas de transporte de carga, conforme pode ser visto em D’Agosto e Ribeiro (2004), Vernimmen et al., (2008), Woxenius e 
Bergqvist, (2011), Leal Jr. e D’Agosto (2011a, 2011b), Feo-Valero et al. (2011), Nealer et al. (2012), Ravibabu (2013), Panagakos et al., (2014), Holmgren et al., (2014), Mohria et al., (2019) e Wolff et al. (2019).

Leal Jr. e D’Agosto (2011a, 2011b) e Ravibabu (2013) descrevem os seguintes atributos associados ao aspecto econômico: custo total do transporte, tempo total de trânsito, confiabilidade e perdas e danos (segurança) como os que mais influenciam a escolha da melhor combinação de alternativas para o transporte de cargas. No caso do transporte de carga por meio da utilização de contêiner, Ravibabu (2013) considera ainda, outros dois atributos, aptidão para contêinerização e urgência. Para Nealer et al (2012), custo e tempo são os atributos econômicos mais importantes. Para Mohria et al., (2019) reduzir o tempo e o custo do transporte para o modo ferroviário com container é um fator determinante para aumentar a sua participação na matriz modal de transporte de carga.

Considerando os aspectos ambientais, a emissão de poluentes atmosféricos e gases de efeito estufa (GEE) são atributos considerados por D’Agosto e Ribeiro (2004), Woxenius e Bergqvist, (2011) e Leal Jr. e D’Agosto (2011a, 2011b), o que ratifica a preocupação com os impactos ambientais locais, regionais e globais no contexto das mudanças climáticas. A emissão de enxofre, por estar associado à formação de material particulado e ser um poluente atmosférico de ação local e regional, é um atributo significativo na indução de mudanças na escolha de alternativas de transporte realizadas em áreas com concentração de população. Em estudos como o de Panagakos et al. (2014) e Holmgren et al. (2014) verificou-se o desempenho de alternativas de transporte frente ao estabelecimento de zonas de baixa emissão de enxofre nas regiões do mar Mediterrâneo, mar do Norte e mar Báltico.

O consumo de energia, um atributo de relevância econômica, quando está associado ao maior custo variável do transporte e ambiental e quanto está associado ao consumo de combustíveis fósseis e emissão de poluentes atmosféricos e GEE, é considerado na totalidade dos trabalhos, sendo citado por Nealer et al., (2012) na avaliação de potenciais políticas relativas a escolha de alternativas de transporte de cargas. Os atributos relacionados ao aspecto ambiental contribuem para estabelecer a imagem das alternativas de transporte sob a ótica da sustentabilidade ambiental, fator qualitativo de decisão mencionado por Vernimmen et al., (2008).

Entretanto, a inclusão do aspecto ambiental nas decisões relacionadas à escolha de alternativas de transporte é contraditória e varia de país para país. Feo-Valero et al. (2011) destacam esse ponto, ao comparar a perspectiva de despachantes de carga espanhóis, que não consideram a redução dos impactos ambientais como relevante para suas decisões quanto a escolha de alternativas de transporte, com a realidade suíça, onde os embarcadores estão dispostos a pagar mais para reduzir emissões de GEE provenientes do transporte de cargas. No que diz respeito a infraestrutura e operação de transporte, Wolff et al. (2019) destacam que as emissões atmosféricas e o número de acidentes são os atributos mais relevantes para avaliações de investimentos e desempenho operacional em transporte de cargas no Brasil.

Para Cazeri et al. (2017) muitos atributos são discutidos nas dimensões econômica e ambiental, no entanto, a dimensão social tem uma lacuna relevante a ser explorada.

Em síntese, para o aspecto econômico, a revisão bibliográfica aponta para os seguintes atributos: (1) custo, (2) tempo, (3) confiabilidade e (4) segurança. No caso do aspecto ambiental, os atributos usualmente considerados são: (1) consumo de energia, (2) poluentes atmosféricos e (3) dióxido de carbono (CO2), sendo este último, o principal GEE. A quantificação destes atributos se faz por meio da identificação de indicadores de desempenho. 


\section{MÉTODO}

De uma forma estruturada, recomenda-se que a avaliação de desempenho das alternativas não usuais para o transporte de produtos de baixo valor agregado siga um procedimento que relacione recursos necessários à operação de transporte com os resultados que se pretende obter. Isto na forma de medidas de desempenho que representem combinações de indicadores de desempenho relacionados a atributos de desempenho representativos dos aspectos econômico e ambiental. As medidas de desempenho obtidas para cada uma das alternativas não usuais para o transporte de produtos de baixo valor agregado devem ser comparadas com as obtidas para as alternativas de transporte especializado com o objetivo de avaliar sua adequação, por meio da garantia dos resultados associados a custo e nível de serviço, sem comprometer o resultado dos atributos de desempenho associados ao aspecto ambiental. Isso pode ser feito por meio do aprimoramento da abordagem integrada que envolve o conceito de eco eficiência (D'Agosto e Ribeiro, 2004) considerando a estrutura paramétrica de aspectos, atributos, indicadores e medidas, apresentada por Leal Jr e D’Agosto (2011a). A Figura 1 sintetiza o procedimento proposto.

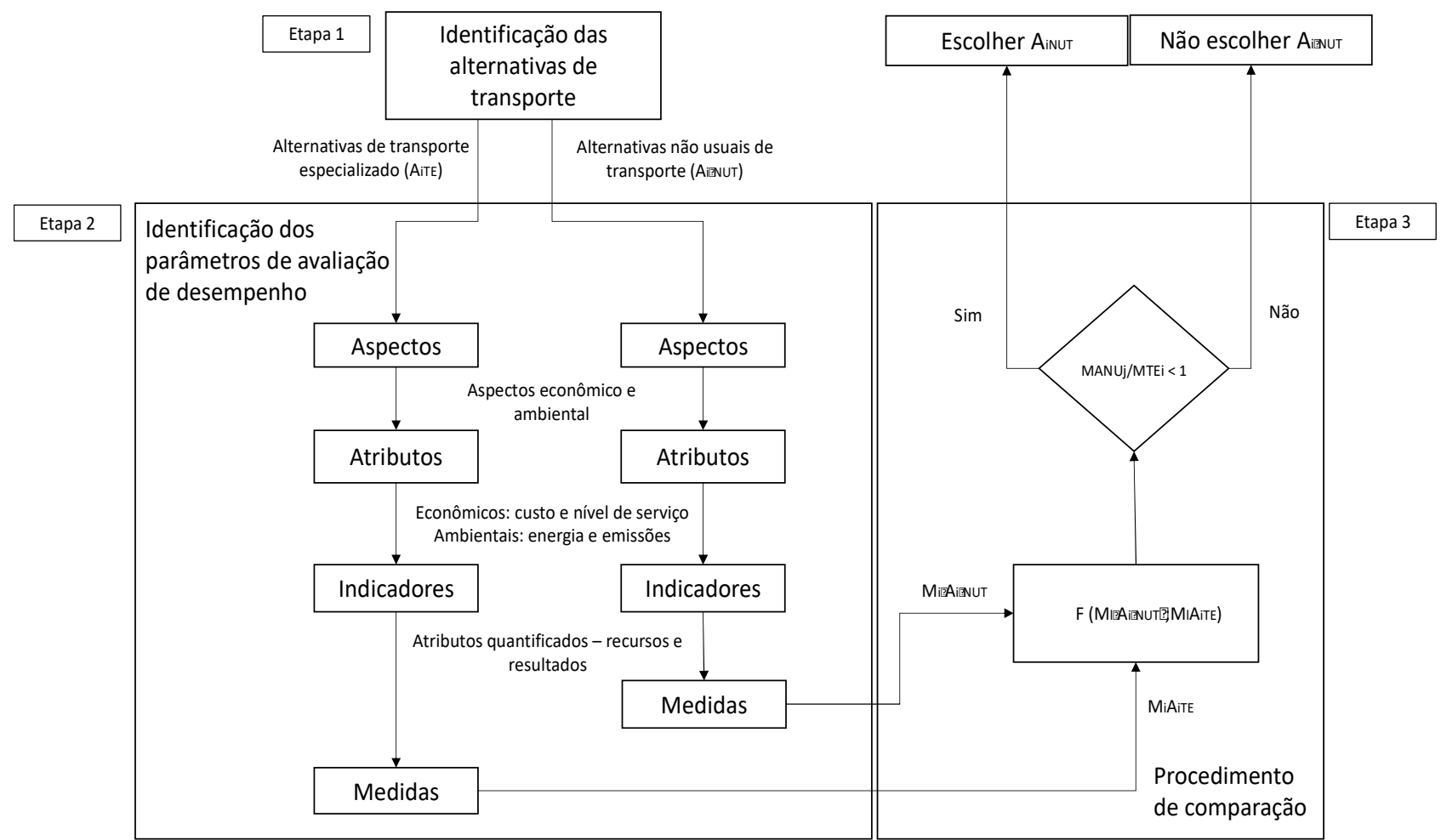

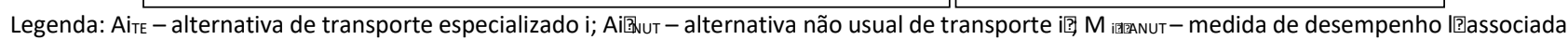
a alternativa não usual de transporte i] MilATE - medida de desempenho I da alternativa de transporte especializado i.

Figura 1. Procedimento para avaliação de desempenho e escolha de alternativas não usuais para o transporte de produtos de baixo valor agregado.

A Etapa 1 do procedimento (Figura 1) considera a identificação das alternativas de transporte. Entende-se que seja possível identificar um conjunto de alternativas relacionadas ao transporte especializado (AiTE - i: 1..n) e outro conjunto de alternativas não usuais para o transporte de carga de baixo valor agregado (Ai'NUT - i': 1..n'). 0 transporte especializado está associado a modos de transporte ou combinação de modos de transporte de grande capacidade onde, por meio da especialização na oferta de transporte é possível obter redução de custo por 
ganho de escala de produção, principal aspecto avaliado para a escolha de alternativas de transporte para este tipo de carga. Já as alternativas não usuais para o transporte de carga de baixo valor agregado consideram o uso de outros modos de transporte ou suas combinações que permitam a oferta de serviço garantindo os mesmos resultados de desempenho para os atributos associados ao aspecto econômico (custo e nível de serviço), sem comprometer o desempenho dos atributos associados ao aspecto ambiental.

Uma vez que AiTE e Ai'NUT tenham sido identificadas, passa-se a Etapa 2 do procedimento, que permitirá a identificação dos parâmetros de avaliação de desempenho de forma estruturada, considerando aspectos, atributos, indicadores e medidas, nesta ordem. Com base na revisão bibliográfica realizada (seção 2 deste artigo) e em função da abordagem conceitual proposta, entende-se que se deva considerar pelo menos os aspectos econômico e ambiental, sendo o primeiro representado pelos atributos custo e nível de serviço e o segundo por, pelo menos, os atributos consumo de energia, emissões de poluentes atmosféricos e gases de efeito estufa.

A identificação das alternativas realizada na Etapa 1 do procedimento determinará os indicadores de desempenho capazes de quantificar os atributos de desempenho, compondo um conjunto de medidas de desempenho específicas (MlAiTE - medida de desempenho l - l: 1..o da alternativa de transporte especializado i; Ml'Ai NUT - medida de desempenho l' - l': 1..o' associada a alternativa não usual de transporte i') que relacionam recursos e resultados para cada alternativa de transporte.

Admitindo que cada uma das alternativas de transporte, especializadas (AiTE) ou não usuais (AínUT), sejam compostas por uma combinação de j (j: 1..p) modos de transporte, que atuam em segmentos de tráfego conectados por k (k: 1..p+1) terminais de transbordo, as Equações 1 a 8 podem ser consideradas para determinação das medidas de desempenho apresentadas na Tabela 1.

Tabela 1 - Medidas de desempenho recomendadas ${ }^{(1)}$.

\begin{tabular}{|c|c|c|c|c|}
\hline \multirow{2}{*}{ Aspectos } & \multirow{2}{*}{ Atributos } & \multicolumn{3}{|l|}{ Medidas $^{(2)}$} \\
\hline & & Denominação & Sigla & Unidade \\
\hline \multirow{4}{*}{ Econômico } & Custo & Custo da alternativa i (i') & $\mathrm{CA}_{\mathrm{i}(\mathrm{i})}$ & $\mathrm{R} \$ / \mathrm{t}$ \\
\hline & NS - Tempo & Tempo médio de transporte da alternativa i (i') & $\mathrm{TA}_{\mathrm{i}\left(\mathrm{i}^{\prime}\right)}$ & h \\
\hline & NS - Confiabilidade & Confiabilidade média da alternativa i (i') & $\operatorname{VTA}_{\mathrm{i}\left(\mathrm{i}^{\prime}\right)}$ & $\%$ \\
\hline & NS - Segurança & Custo com perdas de carga na operação da alternativa i (i') & $\mathrm{CPA}_{\mathrm{i}\left(\mathrm{i}^{\prime}\right)}$ & $\mathrm{R} \$ / \mathrm{t}$ \\
\hline \multirow{3}{*}{ Ambiental } & Consumo de energia & Consumo médio de energia da alternativa i (i') & $C E_{i}\left(i^{\prime}\right)$ & $\mathrm{MJ} / \mathrm{t}$ \\
\hline & \multirow{2}{*}{$\begin{array}{l}\text { Emissão de poluen- } \\
\text { tes atmosféricos e } \\
\text { GEE }\end{array}$} & Emissão média do poluente atmosférico $p$ pela alternativa i (i') & $E P_{i,\left(i^{\prime}\right) p}$ & $\mathrm{~g} / \mathrm{t}$ \\
\hline & & Emissão média de $\mathrm{CO}_{2}$ pela alternativa i (i') & $\mathrm{ECO}_{2 \mathrm{i}(\mathrm{i})}$ & $\mathrm{kg} / \mathrm{t}$ \\
\hline
\end{tabular}

Notas: (1) - as medidas de desempenho recomendadas são consistentes com o resultado da revisão bibliográfica (seção 2 deste artigo); (2) - os indicadores de desempenho que compõe cada uma das medidas de desempenho estão especificados nas Equações (1) a (8) que tem referência nos trabalhos de D'Agosto e Ribeiro, (2004) e Leal Jr e D'Agosto (2011a).

Legenda: NS - Nível de Serviço; GEE - gás de efeito estufa.

$$
C A_{i\left(i^{\prime}\right)}=\frac{\sum_{j=1}^{m} C T_{j} . D_{j}}{V T_{i\left(i^{\prime}\right)}}+\sum_{k=1}^{n} C M_{k}+C O C_{i\left(i^{\prime}\right)}
$$

em que: $\quad C A_{i\left(i^{\prime}\right)} \quad$ Custo da alternativa i ( $\left.i^{\prime}\right)[R \$ / t]$

$\mathrm{CT}_{\mathrm{j}} \quad$ Custo de transporte pelo modo $\mathrm{j}[\mathrm{R} \$ / \mathrm{km}]$

$\mathrm{D}_{\mathrm{j}} \quad$ Distância percorrida pelo modo j [km]

$\mathrm{VT}_{\mathrm{i}(\mathrm{i})} \quad$ Quantidade de produto transportado pela alternativa i (i') [t] 
$\mathrm{CM}_{\mathrm{k}} \quad$ Custo de movimentação no terminal de transbordo $\mathrm{k}[\mathrm{R} \$ / \mathrm{t}]$

$\mathrm{COC}_{\mathrm{i}\left(\mathrm{i}^{\prime}\right)}$ Custo de operação do implemento para a alternativa i (i') [R \$/t]

$$
T A_{i\left(i^{\prime}\right)}=\sum_{j=1}^{m} T M_{j\left(j^{\prime}\right)}+\sum_{k=1}^{n} T N_{k}
$$

em que: $\quad \mathrm{TA}_{\mathrm{i}\left(\mathrm{i}^{\prime}\right)} \quad$ Custo da alternativa i (i') $[\mathrm{R} \$ / \mathrm{t}]$

$\mathrm{TM}_{\mathrm{j}\left(\mathrm{j}^{\prime}\right)} \quad$ Custo de transporte pelo modo $\mathrm{j}[\mathrm{R} \$ / \mathrm{km}]$

$\mathrm{TN}_{\mathrm{k}} \quad$ Distância percorrida pelo modo $\mathrm{j}[\mathrm{km}]$

$$
V T A_{i\left(i^{\prime}\right)}=e_{T A i\left(i^{\prime}\right)}
$$

em que: VTA $\mathrm{V}_{\mathrm{i}\left(\mathrm{i}^{\prime}\right)} \quad$ Variação do tempo médio da alternativa i (i')

el( $\left(^{\prime}\right) \quad$ Desvio bilateral relativo sobre a média de $\mathrm{TA}_{\mathrm{i}}\left(\mathrm{i}^{\mathrm{i}}\right)$, calculado conforme Equação 9.

$$
C P A_{i\left(i^{\prime}\right)}=\left(P C+C A_{i\left(i^{\prime}\right)}\right) \cdot F P_{i\left(i^{\prime}\right)}
$$

em que: $\quad \mathrm{CPA}_{\mathrm{i}\left(\mathrm{i}^{\prime}\right)} \quad$ Custo da alternativa i ( $\left.\mathrm{i}^{\prime}\right)[\mathrm{R} \$ \mathrm{t}]$

$\mathrm{FP}_{\mathrm{i}(\mathrm{i})} \quad$ Custo de transporte pelo modo $\mathrm{j}[\mathrm{R} \$ / \mathrm{km}]$

PC Distância percorrida pelo modo $\mathrm{j}[\mathrm{km}]$

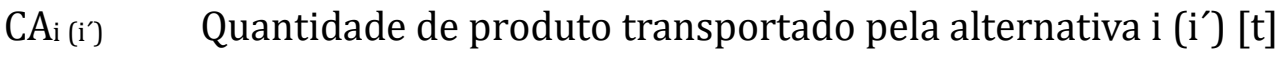

$$
C F_{i\left(i^{\prime}\right)}=\frac{\sum_{j=1}^{m} F C C F T_{j} \cdot D_{j}}{V T_{i\left(i^{\prime}\right)}}+\sum_{k=1}^{n} F C C F N_{k}
$$

em que: $\quad \mathrm{CF}_{\mathrm{i}\left(\mathrm{i}^{\top}\right)} \quad$ Consumo médio de combustível fóssil pela alternativa i ( $\left.\mathrm{i}^{\prime}\right)[\mathrm{l} / \mathrm{t}]$

FCCFT $_{\mathrm{j}} \quad$ Fator de consumo de combustível fóssil pelo modo de transporte $\mathrm{j}[\mathrm{l} / \mathrm{km}]$

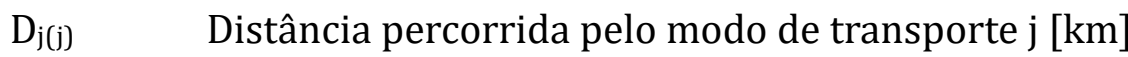

$\mathrm{VT}_{\mathrm{i}\left(\mathrm{i}^{\prime}\right)} \quad$ Quantidade de produto transportado pela alternativa i (i') [t]

FCCFN $_{\mathrm{k}} \quad$ Fator de consumo de combustível fóssil para movimentação no nó k [l/t]

$$
C E_{i\left(i^{\prime}\right)}=C F_{i\left(i^{\prime}\right)} F C E C F+\sum_{k=1}^{n} C E C T_{k}
$$

em que: $\quad \mathrm{CE}_{\mathrm{i}\left(\mathrm{i}^{\prime}\right)} \quad$ Consumo energético médio da alternativa i ( $\left.\mathrm{i}^{\prime}\right)[\mathrm{MJ} / \mathrm{t}]$

FCECF Fator de conteúdo energético do combustível fóssil [MJ $/ \mathrm{kg}]$

$\mathrm{CECT}_{\mathrm{k}}$ Consumo energético médio de movimentação no terminal $\mathrm{k}[\mathrm{MJ} / \mathrm{t}]$

$$
E P_{i\left(i^{\prime}\right), p}=\frac{\sum_{j=1}^{m} F E T_{P, j} \cdot D_{j}}{V T_{i\left(i^{\prime}\right)}}+\sum_{k=1}^{n} F E M_{P, k}
$$

em que: $\quad \mathrm{EP}_{\mathrm{i}\left(\mathrm{i}^{\prime}\right) \mathrm{p}} \quad$ Emissão do poluente atmosférico p pela alternativa i (i') [g/t $]$

FET $_{\mathrm{p}, \mathrm{j}} \quad$ Fator de emissão do poluente atmosférico $\mathrm{p}$ pelo modo de transporte $\mathrm{j}[\mathrm{g} / \mathrm{km}]$

$\mathrm{D}_{\mathrm{j}} \quad$ Distância percorrida pelo modo de transporte $\mathrm{j}[\mathrm{km}]$

$\mathrm{VT}_{\mathrm{i}(\mathrm{i})}$ Quantidade de produto transportado pela alternativa i (i') [t]

$\mathrm{FEM}_{\mathrm{p}, \mathrm{k}} \quad$ Fator de emissão do poluente atmosférico p para movimentação no nó $\mathrm{k}[\mathrm{g} / \mathrm{t}]$

$\mathrm{p} \quad$ Poluente atmosférico (p: CO, NMHC, NO $\mathrm{N}_{\mathrm{x}} \mathrm{MP}$ )

$$
\mathrm{ECO}_{2 i\left(i^{\prime}\right)}=\mathrm{CF}_{i\left(i^{\prime}\right)} \mathrm{FCO}_{2}
$$


em que: $\quad \mathrm{ECO}_{2 \mathrm{i}\left(\mathrm{i}^{\prime}\right)} \quad$ Emissão média de $\mathrm{CO}_{2}$ pela alternativa i (i') $[\mathrm{kg} / \mathrm{t}]$

$\mathrm{FCO}_{2} \quad$ Fator de emissão de $\mathrm{CO}_{2}$ para o combustível fóssil [kg/l]

Para todas as medidas ( $\mathrm{l} \mathrm{e} \mathrm{l}^{\prime}$ ) onde houver a necessidade de tratamento estatístico considerase que estas seguem uma função densidade de probabilidade normal, identificando-se os valores e, t, n-1 e $\alpha / 2$ (Equação 9), sendo essa hipótese testada por meio do teste Kolmogorov-Smirnov.

$$
e_{l\left(l^{\prime}\right)}=t_{n_{l\left(l^{\prime}\right)}-1, \alpha / 2} \cdot \frac{S_{l\left(l^{\prime}\right)}}{\sqrt{n_{l\left(l^{\prime}\right)}-1}}
$$

em que: el(1') Desvio bilateral relativo sobre a média da medida de desempenho l(l')

$\operatorname{tn}_{n i\left(l^{\prime}\right)-1, \alpha / 2}$ Valor da função de distribuição de probabilidade t de Student-bicaudal para os parâmetros n-1 e $\alpha / 2$

SI(I') Desvio padrão da medida de desempenho $1\left(\mathrm{l}^{\prime}\right)$

$\mathrm{n}_{\mathrm{l}\left(\mathrm{I}^{\prime}\right)} \quad$ Tamanho da amostra da medida de desempenho $\mathrm{l}^{\left(\mathrm{l}^{\prime}\right)}$

$\alpha / 2 \quad$ Índice de significância estatística da amostra

Escolher medidas de desempenho que apresentem comportamento simétrico, variando na mesma direção ("quanto menor melhor" ou "quanto maior melhor"), permite um tratamento homogêneo dos dados que usualmente apresentam ordem de grandeza variada e devem ser normalizados para comparação de forma isolada ou agregada em uma mesma base de medida. Por simplicidade, para a normalização das medidas de desempenho sugere-se a Equação 10, para o caso de "quanto menor melhor" e a Equação 11 para o caso de "quanto maior melhor".

$$
M N_{i\left(i^{\prime}\right) l\left(l^{\prime}\right)}=\frac{M_{i\left(i^{\prime}\right) l\left(l^{\prime}\right) \min }}{M_{i\left(i^{\prime}\right) l\left(l^{\prime}\right)}}
$$

em que: $\quad \mathrm{MN}_{\mathrm{i}\left(\mathrm{i}^{\prime}\right) \mathrm{l}\left(\mathrm{I}^{\prime}\right)} \quad$ Resultado normalizado da medida $\left.\mathrm{l}^{\prime} \mathrm{l}^{\prime}\right)$ para a alternativa $\mathrm{i}^{\left(\mathrm{i}^{\prime}\right) \text {; }}$

$\mathrm{M}_{\mathrm{i}\left(\mathrm{i}^{\prime}\right)\left(\mathrm{I}^{\prime}\right) \min }$ Menor valor da medida $\left.\mathrm{l}^{\prime} \mathrm{l}^{\prime}\right)$ para a alternativa $\mathrm{i}\left(\mathrm{i}^{\prime}\right)$;

$\mathrm{M}_{\mathrm{i}(\mathrm{i}) l\left(\mathrm{I}^{\prime}\right)} \quad$ Valor da medida $\mathrm{l}(\mathrm{l})$ para a alternativa $\mathrm{i}\left(\mathrm{i}^{\prime}\right)$.

$$
M N_{i\left(i^{\prime}\right) l\left(l^{\prime}\right)}=\frac{M_{i\left(i^{\prime}\right) l\left(l^{\prime}\right)}}{M_{i\left(i^{\prime}\right) l\left(l^{\prime}\right) \max }}
$$

em que: $\quad \mathrm{MN}_{\mathrm{i}\left(\mathrm{i}^{\prime}\right) \mathrm{l}\left(\mathrm{I}^{\prime}\right)} \quad$ Resultado normalizado da medida $\left(\mathrm{l}^{\prime}\right)$ para a alternativa $\mathrm{i}^{\left(\mathrm{i}^{\prime}\right) \text {; }}$

$\mathrm{M}_{\mathrm{i}\left(\mathrm{i}^{\prime}\right) l\left(\mathrm{I}^{\prime}\right) \max }$ Maior valor da medida $\mathrm{l}^{\left(\mathrm{l}^{\prime}\right)}$ para a alternativa $\mathrm{i}\left(\mathrm{i}^{\prime}\right)$;

$\mathrm{M}_{\mathrm{i}(\mathrm{i}) \mathrm{l}\left(\mathrm{I}^{\prime}\right)} \quad$ Valor da medida $\mathrm{l}(\mathrm{l})$ para a alternativa $\mathrm{i}\left(\mathrm{i}^{\prime}\right)$.

A partir dos resultados obtidos por meio das Equações 10 e 11, é possível estabelecer comparação isolada entre as medidas de desempenho normalizadas $\mathrm{MN}_{\mathrm{l}} \mathrm{A}_{\mathrm{i}}{ }^{\prime} \mathrm{NUT}$, associadas a alternativa não usual de transporte e as medidas de desempenho normalizadas $\mathrm{MN}_{1} \mathrm{~A}_{\mathrm{iTE}}$, associadas a alternativa de transporte especializado, considerando esta última como referencial para avaliação (Equação 12).

$$
\frac{M N_{l^{\prime}} A_{i^{\prime} N U T}}{M N_{l} A_{i T E}}>1 \text {; então, MNl' } \mathrm{A}_{\mathrm{i}^{\prime} \mathrm{NUT}} \text { melhor que } \mathrm{MN}_{\mathrm{l}} \mathrm{A}_{\mathrm{iTE}}
$$

Estabelecendo uma das Aite como referência para avaliação de cada uma das Ai'NUT, a comparação isolada entre as medidas de desempenho normalizadas $\mathrm{MN}^{\prime} \mathrm{A}_{\mathrm{i}}{ }^{\prime} \mathrm{NUT}$, associadas a alternativa não usual de transporte e as medidas de desempenho normalizadas $\mathrm{MN}_{\mathrm{l}} \mathrm{A}_{\mathrm{iTE}}$, associadas a alternativa de transporte especializado pode ser obtida por meio da Equação 13.

$$
\frac{M N_{l^{\prime}} A_{i^{\prime} N U T}}{M N_{l} A_{i T E}}>1 \text {; então, } M N_{l^{\prime}} A_{i^{\prime} N U T} \text { melhor que MN } N_{1} A_{i T E}
$$


Os resultados obtidos por meio da Equação 13 também permitem que se estabeleça uma hierarquia entre as alternativas não usuais de transporte e as alternativas de transporte especializado. Caso se deseje realizar um teste de sensibilidade, é possível introduzir pesos, variando de 0 a 1, para as medidas de desempenho, de forma a valorizar o aspecto econômico ou ambiental.

Indicadores associados a medida de custo podem ser obtidos a partir de consulta de mercado (cotação), representando o valor mais provável para uma determinada alternativa. Já os indicadores associados as medidas de nível de serviço que envolvem tempo (média e sua variabilidade), devem ser obtidos por meio de um levantamento de campo, para um horizonte de tempo de coleta de dados que garanta a significância estatística esperada pela equipe que está aplicando o procedimento. Isso também se aplica aos indicadores associados a custo com perdas e consumo de energia.

É desejável que a quantificação dos indicadores que compõe as medidas de emissão de poluentes atmosféricos e GEE derive de levantamento de campo e neste caso os resultados refletiriam de forma mais próxima à realidade. Fazer isto usualmente exige o monitoramento dos equipamentos com dispositivos complexos, o que resulta em uma experiência não trivial e cara. Assim sendo, o valor destes indicadores pode ser estimado por meio de fatores médios relacionados ao consumo de energia ou a distância percorrida, oriundos de inventários de emissão nacionais ou regionais. No Brasil já foram realizados 2 inventários nacionais de emissão de poluentes atmosféricos por veículos rodoviários (em 2011 e 2013) e 1 por transporte ferroviário (2012), o que aproxima os valores estimados da realidade nacional e minimiza a necessidade de adaptações e simplificações.

\section{APLICAÇÃO - O CASO DO CALCÁRIO SIDERÚRGICO}

O procedimento para avaliação de desempenho e escolha de alternativas não usuais para o transporte de produtos de baixo valor agregado será aplicado ao transporte de calcário para uma indústria siderúrgica específica localizada na região Sudeste do Brasil.

\subsection{Etapa 1 - Identificação das alternativas de transporte}

Para a produção de uma tonelada de aço são necessárias aproximadamente 2,7 toneladas de insumos, destacando-se o minério de ferro, o carvão e o calcário. Este último, embora imprescindível para a fabricação do aço, representa uma fração de 0,04\% na sua composição (IAB, 2011), situação que o coloca em posição desfavorável perante o minério de ferro e o carvão siderúrgico, na competição pela oferta de transporte especializado. Por outro lado, o calcário caracteriza-se por possuir baixo custo de aquisição, isso faz do transporte um dos itens mais onerosos e importantes como componente do custo final deste produto.

Para a distribuição de insumos siderúrgicos no Brasil, o modo ferroviário corresponde a $69 \%$, o rodoviário a 29\% e o hidroviário a 2\% (IAB, 2011). No entanto, para o transporte do calcário, o modo rodoviário, que apresenta os maiores custos e impactos ambientais, é o mais utilizado, seguido pelo ferroviário e o hidroviário, este último utilizado de forma combinada, juntamente com outros modos de transportes terrestres (rodoviário e ferroviário) (Poso, 2007; CNT, 2011; Coelho Neto, 2006 e Castro, 2002).

Nesse contexto, torna-se necessário identificar e avaliar o desempenho de alternativas não usuais para o transporte de produtos de baixo valor agregado, que tragam benefícios econômicos e ambientais ao transporte de insumos, dentro do processo produtivo do aço. Desde que 
haja disponibilidade de equipamentos (vagões plataforma e contêineres), uma alternativa possível é o emprego do transporte intermodal rodoferroviário com uso de contêineres para suprimento de calcário siderúrgico, alternativa não usual de transporte que teve sua prática experimental iniciada em 2008 por uma das principais produtoras de aço do Brasil, a ArcellorMittal Tubarão (AMT) (Santos, 2012).

O calcário é um material composto por inúmeras partículas de rocha, caracterizando-o como uma carga tipo granel que é transportado em lotes de grande volume e peso. Tomando como base a localização dos principais fornecedores de calcário na região metropolitana de Belo Horizonte, a alternativa ( $\mathrm{A}_{1 \mathrm{TE}}$ ) de transporte especializado (Figura 2 e Tabela 2 ) utiliza conjuntos compostos por caminhão trator 6x2 e semirreboque caçamba basculante de 3 eixos e $30 \mathrm{~m}^{3}$ de capacidade com peso bruto total (PBT) de 23 t que fazem o transporte do calcário da mina (0) por $52 \mathrm{~km}$ de rodovia até o terminal ferroviário de Santa Luzia (T1) em Minas Gerais onde o produto é basculhado e vagões gôndola são carregados por pás carregadeiras. 0 calcário é transportado por $652 \mathrm{~km}$ de ferrovia (Estrada de Ferro Vitória Minas - EFVM) até o Terminal de Serra (T2) no Espírito Santo, onde viradores basculham o calcário nas esteiras transportadoras que alimentam silos ou direcionam o produto para pátios completando o percurso (4 km) até a unidade de calcinação (D).

Em função das limitações de oferta de transporte ferroviário especializado para o transporte de calcário, a AMT optou por três alternativas não usuais para o transporte de calcário (Figura 2, Tabela 2). Na primeira alternativa, foi preciso substituir $12 \mathrm{~km}$ de transporte ferroviário por $26 \mathrm{~km}$ de transporte rodoviário (conjuntos compostos por caminhão trator 6x2 e semirreboque caçamba basculante de 3 eixos e $30 \mathrm{~m}^{3}$ de capacidade com peso bruto total (PBT) de $23 \mathrm{t}$ ) em segmento próximo ao terminal de destino, trocando o Terminal de Serra pelo Terminal de Cariacica (T3) e compondo uma alternativa de transporte do tipo rodo-ferro-rodo (A1NUT).

A segunda alternativa utiliza o transporte intermodal rodoferroviário na forma rodo-ferrorodo (A2NUT) com utilização de contêineres e foi adotada com o objetivo de reduzir a degradação da carga transportada uma vez que todo o produto é movimentado dentro de um recipiente fechado. Em A2NuT o calcário é carregado em contêineres de 20 pés por meio de recuperadores. Os contêineres são carregados em conjuntos de PBT de $23 \mathrm{t}$ formados por caminhão trator $6 \mathrm{x} 2$ e semirreboque plataforma que trafegam por $23 \mathrm{~km}$ de rodovia até o Terminal de Confins (T4) em Minas Gerais, onde empilhadeiras (reach stackers) transferem os contêineres para vagões plataforma. A composição ferroviária trafega por $670 \mathrm{~km}$ até o Terminal de Serra (T2) no Espírito Santo onde os contêineres são novamente transferidos para conjuntos de PBT de $23 \mathrm{t}$ formados por caminhão trator $6 \times 2$ e semirreboque plataforma que trafegam por $3 \mathrm{~km}$ de rodovia até a até a unidade de calcinação (D), onde o produto é basculhado.

A terceira alternativa (A3NUT) utiliza integralmente o modo rodoviário (pela rodovia BR262) por meio de conjuntos compostos por caminhão trator 6x2 e semirreboque caçamba basculante de 3 eixos e $30 \mathrm{~m} 3$ de capacidade com peso bruto total (PBT) de $23 \mathrm{t}$, que ligam a mina (O) em Belo Horizonte ao pátio (P) do Porto de Tubarão no Espírito Santo e esteiras transportadoras para fazer a entrega final $(2 \mathrm{~km})$ para a unidade de calcinação. 


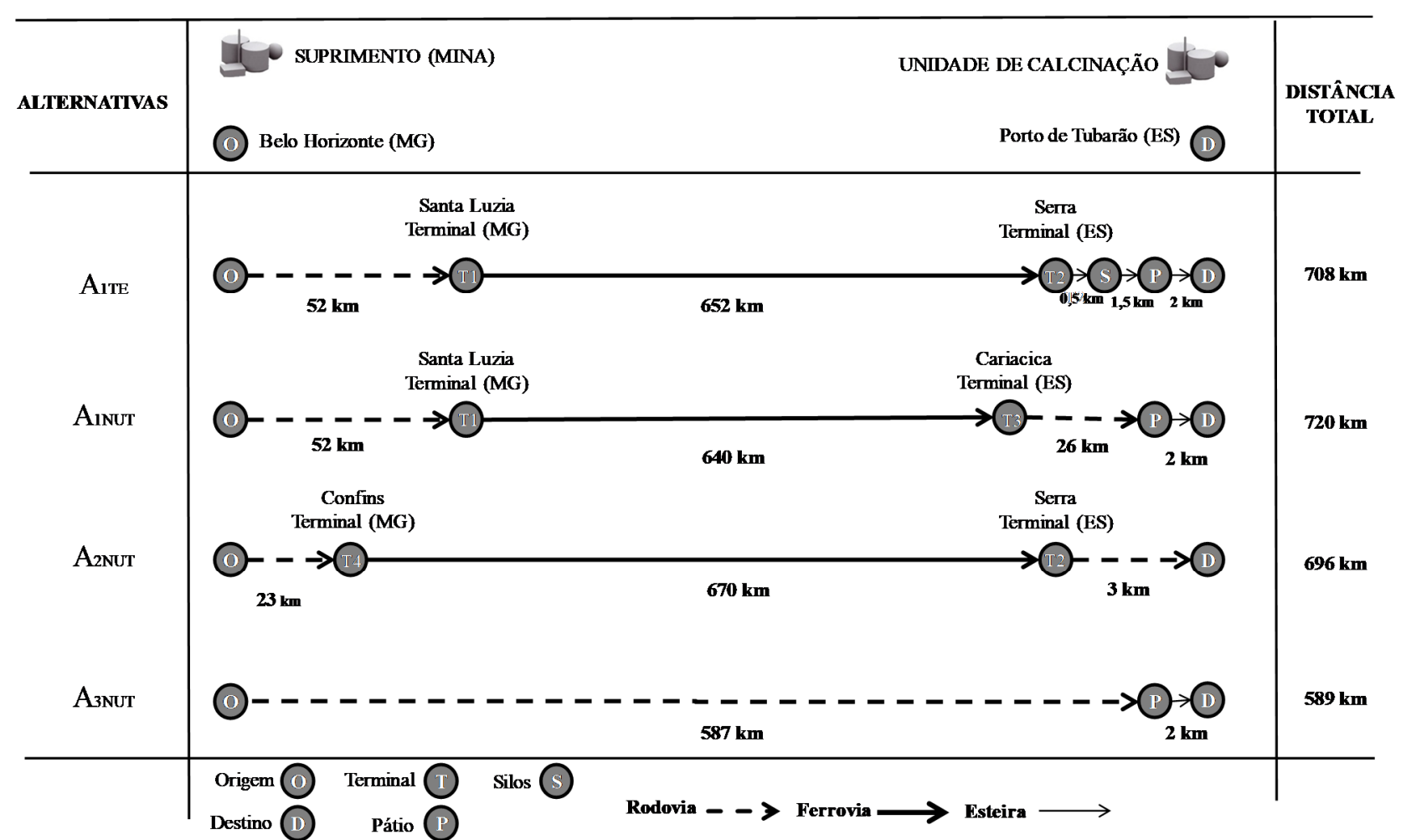

Notas: Para todas as alternativas o transporte se dá entre a mesma origem (O) e o mesmo destino (D).

Legenda: $\mathrm{A}_{1 \mathrm{TE}}$ - alternativa de transporte especializado do tipo rodo-ferro; $\mathrm{A}_{1 \mathrm{NUT}}$ - alternativa de transporte não usual do tipo rodo-ferro-rodo; $\mathrm{A}_{2 \mathrm{NUT}}$ - alternativa de transporte não usual do tipo rodo-ferro-rodo com container e $A_{3 N U T}$ - alternativa de transporte não usual do tipo rodoviário.

Figura 2. Diagramas ilustrativos (sem escala) das alternativas de transporte de calcário.

Tabela 2 - Características complementares das alternativas para transporte de calcário(1)

\begin{tabular}{ccccccc}
\hline \multirow{2}{*}{ Alternativas } & \multicolumn{4}{c}{ Divisão modal(2) } & \multicolumn{2}{c}{ Terminais de transbordo } \\
\cline { 2 - 6 } & Rodoviário & Ferroviário & Esteira & Total & Número & Equipamento \\
\hline $\mathrm{A}_{1 \mathrm{TE}}$ & $7,3 \%$ & $92,1 \%$ & $0,6 \%$ & $100 \%$ & 4 & Pá carregadeira \\
$\mathrm{A}_{1 \mathrm{NUT}}$ & $10,8 \%$ & $88,9 \%$ & $0,3 \%$ & $100 \%$ & 3 & Pá carregadeira \\
$\mathrm{A}_{\text {2NUT }}$ & $3,7 \%$ & $96,3 \%$ & $0,0 \%$ & $100 \%$ & 2 & Empilhadeira de contêiner \\
$\mathrm{A}_{3 \mathrm{NUT}}$ & $99,7 \%$ & $0,0 \%$ & $0,3 \%$ & $100 \%$ & 1 & Pá carregadeira \\
\hline
\end{tabular}

Nota: (1) - informações complementares aos dados apresentados na Figura 2; (2) em percentual da distância.

Legenda: $\mathrm{A}_{1 \mathrm{TE}}$ - alternativa de transporte especializado do tipo rodo-ferro; $\mathrm{A}_{1 \mathrm{~N} U \mathrm{~T}}$ - alternativa de transporte não usual do tipo rodo-ferro-rodo; $\mathrm{A}_{2 \mathrm{NUT}}$ - alternativa de transporte não usual do tipo rodo-ferro-rodo com container e $\mathrm{A}_{3 \mathrm{NUT}}$ - alternativa de transporte não usual do tipo rodoviário.

\subsection{Etapa $\mathbf{2}$ - Identificação dos parâmetros para avaliação de desempenho}

Para atender aos objetivos deste artigo e garantir a escolha de alternativas não usuais para $o$ transporte de calcário que apresentem custos e níveis de serviço iguais ou melhores ao que se obteria por meio do transporte especializado, sem comprometer o desempenho ambiental, a avaliação de desempenho das alternativas de transporte deve considerar aspectos econômicos e ambientais.

Como foi apresentado nas seções 2 e 3 deste artigo, a revisão bibliográfica aponta para a escolha dos atributos e medidas de desempenho apresentados na Tabela 1, que foram efetivamente considerados por meio da combinação de indicadores de desempenho conforme Equações de 1 a 8.

Os valores de tempo de operação e de consumo de energia, na forma de combustível (óleo diesel) para as locomotivas e caminhões e energia elétrica para as correias transportadoras, 
foram obtidos pelo acompanhamento da operação em campo ao longo do ano de 2014. Os valores de custo foram obtidos por meio de consulta ao mercado. As perdas foram estimadas pela diferença entre a massa de produto embarcado na origem e recebido no destino final (unidade de calcinação), considerando a operação ao longo de todo o ano de 2014. Os resultados são apresentados na Tabela 3.

Os valores oriundos de levantamento estatístico apresentaram intervalo de variação de +$5 \%$ sobre a média, após utilização da Equação 9. Estes atributos são considerados como os recursos consumidos para produzir como resultado o transporte de uma tonelada de calcário da origem (mina) até o destino (unidade de calcinação), conforme apresentado na Figura 2.

Tabela 3 - Valores dos indicadores de desempenho coletados em campo

\begin{tabular}{|c|c|c|c|c|c|c|c|c|c|c|c|c|c|}
\hline & & & & & & & & & & Terminal & & & \\
\hline & \multicolumn{3}{|c|}{ Caminhão } & \multicolumn{3}{|c|}{ Locomotiva } & \multicolumn{3}{|c|}{ Correia Transportadora } & \multicolumn{2}{|c|}{ Pá Carregadeira } & \multicolumn{2}{|c|}{ Empilhadeira de contêine } \\
\hline & $\begin{array}{l}\text { Custo } \\
{[R \$ / t]}\end{array}$ & Tempo (h) & $\begin{array}{c}\text { Energia } \\
{\left[I_{\text {diesel }} /\right.} \\
\text { t transpostada }\end{array}$ & $\begin{array}{l}\text { Custo } \\
\text { [R\$/t] }\end{array}$ & Tempo (h) & $\begin{array}{c}\text { Energia } \\
{\left[I_{\text {diesel }} /\right.} \\
\left.\mathbf{t}_{\text {transpostada }}\right]\end{array}$ & Custo $[R \$ / t]$ & Tempo (h) & $\begin{array}{c}\text { Energia } \\
{\left[I_{\text {diesel }} /\right.} \\
\mathbf{t}_{\text {transpostada] }}\end{array}$ & Tempo (h) & $\begin{array}{c}\text { Energia } \\
{\left[I_{\text {diesel }} /\right.} \\
\left.\mathbf{t}_{\text {transpostada }}\right]\end{array}$ & Tempo (h) & $\begin{array}{c}\text { Energia } \\
{\left[I_{\text {diesel }} /\right.} \\
\left.t_{\text {transportada }}\right]\end{array}$ \\
\hline $\mathrm{A}_{\mathrm{ITE}}$ & 8,42 & 1,50 & 0,57 & 47,66 & 109,48 & 2,78 & 14,89 & 0,04 & 0,32 & 1,44 & 0,12 & 0,00 & 0,00 \\
\hline $\mathrm{A}_{\mathrm{INUT}}$ & 13,43 & 1,50 & 0,68 & 46,60 & 99,58 & 2,74 & 16,28 & 0,02 & 0,60 & 2,09 & 0,12 & 0,00 & 0,00 \\
\hline $\mathrm{A}_{2 \mathrm{NUT}}$ & 6,01 & 1,77 & 0,16 & 39,33 & 66,81 & 2,51 & 20,25 & 0,00 & 0,00 & 0,00 & 0,00 & 1,08 & 0,47 \\
\hline $\mathrm{A}_{3 \mathrm{NUT}}$ & 65,83 & 19,81 & 6,39 & 0,00 & 0,00 & 0,00 & 2,24 & 0,02 & 0,60 & 0,69 & 0,28 & 0,00 & 0,00 \\
\hline
\end{tabular}

Legenda: A1TE - alternativa de transporte especializado do tipo rodo-ferro; A1NUT - alternativa de transporte não usual do tipo rodo-ferro-rodo; A2NUT - alternativa de transporte não usual do tipo rodo-ferro-rodo com container e A3NUT - alternativa de transporte não usual do tipo rodoviário.

Os poluentes atmosféricos regulamentados no Brasil e para os quais existe estimativa dos fatores de emissões são o monóxido de carbono (CO), os hidrocarbonetos não metano (HCNM), os óxidos de nitrogênio ( $\mathrm{NO}_{2}$ e $\mathrm{NO}_{3}$, considerados como $\mathrm{NO}_{\mathrm{x}}$ ) e o material particulado (MP) (MMA, 2011).

Para os veículos tipo caminhão pesado (conjunto caminhão trator e semirreboque de 3 eixos), utilizados no modo rodoviário, os fatores de emissão dos poluentes atmosféricos e do dióxido de carbono $\left(\mathrm{CO}_{2}\right)$ foram obtidos a partir de MMA (2011). Para o modo ferroviário (locomotiva de manobra e locomotiva de tração), tais informações, foram obtidas a partir de Lewis et al. (2009) (Tabela 4).

Tabela 4 - Fatores de emissão de poluentes atmosféricos e emissão de $\mathrm{CO}_{2}$

\begin{tabular}{|c|c|c|c|c|c|}
\hline Equipamento & $\begin{array}{c}\mathrm{CO}_{2} \\
{\left[\mathrm{~kg} / \mathrm{l}_{\text {diesel }}\right]}\end{array}$ & $\begin{array}{c}\text { CO } \\
\text { [g poluente } / \mathrm{km} \text { ] }\end{array}$ & $\begin{array}{c}\text { HCNM } \\
\text { [gpoluente/ } / \mathrm{km} \text { ] }\end{array}$ & 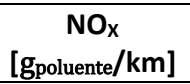 & $\begin{array}{c}\text { MP } \\
\text { [g goluente } / \mathrm{km} \text { ] }\end{array}$ \\
\hline Caminhão pesado & 2,671 & 4,05 & 1,38 & 29,33 & 0,57 \\
\hline Pá carregadeira & 2,671 & 4,05 & 1,38 & 29,33 & 0,57 \\
\hline Empilhadeira contêiner & 2,671 & 4,05 & 1,38 & 29,33 & 0,57 \\
\hline Locomotiva manobra & 2,671 & 26,00 & 6,80 & 74,00 & 3,40 \\
\hline Locomotiva tração & 2,671 & 28,00 & 7,00 & 104,00 & 8,40 \\
\hline
\end{tabular}

Para a movimentação do calcário nos terminais considerou-se para os equipamentos de pátio (pá carregadeira ou empilhadeiras de contêiner) os fatores de emissão de poluentes atmosféricos e de emissão de dióxido de carbono $\left(\mathrm{CO}_{2}\right)$ semelhantes aos de um caminhão pesado. Quanto às correias transportadoras, estas utilizam energia elétrica gerada a partir de fonte hidráulica para sua movimentação e nenhuma emissão de poluentes atmosféricos por uso final foi considerada em sua operação. 
Os fatores de conteúdo energético e de emissão de $\mathrm{CO}_{2}$ considerados para o óleo diesel foram de 40,87 MJ/l e 2,671 kg/l, respectivamente (MMA, 2011).

\subsection{Etapa 3 - Procedimento de comparação}

As medidas de desempenho escolhidas apresentam comportamento simétrico, variando na mesma direção ("quanto menor melhor"), permitindo que se use a Equação 10 para o processo de normalização e as Equação 12 e 13 para a realização da comparação das medidas de desempenho de forma isolada e agregada, respectivamente. A seção 5 apresenta os resultados obtidos e sua discussão.

\section{RESULTADOS E DISCUSSÃO}

A Tabela 5 apresenta os valores obtidos para as medidas de desempenho conforme estabelecido no item 3 por meio do conjunto de Equações de 1 a 8.

Tabela 5 - Valores das medidas e desempenho para cada uma das alternativas

\begin{tabular}{|c|c|c|c|c|c|c|c|c|c|c|}
\hline \multirow{2}{*}{$\frac{\text { Aspectos }}{\text { Medidas }}$} & \multicolumn{4}{|c|}{ Econômico } & \multicolumn{6}{|c|}{ Ambiental } \\
\hline & $\begin{array}{c}\mathrm{CA} \\
{[\mathrm{R} \$ / \mathrm{t}]}\end{array}$ & $\begin{array}{l}\text { TA } \\
{[\mathrm{h}]}\end{array}$ & VTA & $\begin{array}{c}\mathrm{CPA} \\
{[\mathrm{R} \$ / \mathrm{t}]}\end{array}$ & $\begin{array}{c}\mathrm{CE} \\
{[\mathrm{MJ} / \mathrm{t}]}\end{array}$ & $\begin{array}{l}\text { EPco } \\
{[\mathrm{g} / \mathrm{t}]}\end{array}$ & $\begin{array}{c}\text { EPнсNм } \\
{[\mathrm{g} / \mathrm{t}]}\end{array}$ & $\begin{array}{l}E P_{\mathrm{NOx}} \\
{[\mathrm{g} / \mathrm{t}]}\end{array}$ & $\begin{array}{l}\text { EPMP } \\
{[g / t]}\end{array}$ & $\begin{array}{c}\text { Eco2 } \\
{[\mathrm{kg} / \mathrm{t}]}\end{array}$ \\
\hline $\mathrm{A}_{1 \mathrm{TE}}$ & 70,97 & 112,46 & 0,09 & 33,22 & 126,82 & 22,30 & 5,88 & 92,58 & 5,70 & 9,38 \\
\hline $\mathrm{A}_{1 \mathrm{NUT}}$ & 76,31 & 103,19 & 0,09 & 26,20 & 139,43 & 23,62 & 6,32 & 101,29 & 5,83 & 10,32 \\
\hline $\mathrm{A}_{2 \mathrm{NUT}}$ & 65,59 & 69,66 & 0,03 & 5,91 & 113,06 & 20,98 & 5,43 & 84,50 & 5,60 & 8,39 \\
\hline$A_{3 N U T}$ & 68,07 & 20,52 & 0,04 & 9,08 & 240,24 & 22,99 & 7,83 & 166,51 & 3,24 & 17,80 \\
\hline
\end{tabular}

Legenda: $\mathrm{A}_{1 \mathrm{TE}}$ - alternativa de transporte especializado do tipo rodo-ferro; $\mathrm{A}_{1 \mathrm{NUT}}$ - alternativa de transporte não usual do tipo rodo-ferrorodo; $A_{2 N U T}$ - alternativa de transporte não usual do tipo rodo-ferro-rodo com container e A3NuT - alternativa de transporte não usual do tipo rodoviário. CA - custo médio de transporte da alternativa; TA - tempo médio de transporte da alternativa; VTA - confiabilidade da alternativa; CPA - custo médio com perdas de carga na operação da alternativa; CE - consumo médio de energia da alternativa; EP - emissão média do poluente atmosférico pela alternativa; ECO - emissão média de $\mathrm{CO}_{2}$ pela alternativa.

A normalização dos valores apresentados na Tabela 5, por meio da aplicação da Equação 10, leva aos resultados apresentados na Tabela 6.

Tabela 6 - Valores normalizados das medidas e desempenho para cada uma das alternativas

\begin{tabular}{ccccccccccc}
\hline Aspectos & \multicolumn{4}{c}{ Econômico } & \multicolumn{9}{c}{ Ambiental } \\
\hline Medidas & CA & TA & VTA & CPA & CE & EPCO & EPHCNM & EPNOx & EPMP & EPCO2 \\
\hline A1TE & 0,924 & 0,111 & 0,333 & 0,178 & 0,891 & 0,941 & 0,923 & 0,913 & 0,568 & 0,894 \\
\hline A1NUT & 0,860 & 0,121 & 0,312 & 0,226 & 0,811 & 0,888 & 0,859 & 0,834 & 0,556 & 0,813 \\
\hline A2NUT & 1,000 & 0,180 & 1,000 & 1,000 & 1,000 & 1,000 & 1,000 & 1,000 & 0,579 & 1,000 \\
\hline A3NUT & 0,964 & 1,000 & 0,744 & 0,651 & 0,471 & 0,913 & 0,693 & 0,507 & 1,000 & 0,471 \\
\hline
\end{tabular}

Legenda: $\mathrm{A}_{1 \mathrm{TE}}$ - alternativa de transporte especializado do tipo rodo-ferro; $\mathrm{A}_{1 \mathrm{NUT}}$ - alternativa de transporte não usual do tipo rodo-ferro-rodo; $\mathrm{A}_{2 \mathrm{NUT}}$ - alternativa de transporte não usual do tipo rodo-ferro-rodo com container e A3NUT - alternativa de transporte não usual do tipo rodoviário. CA - custo médio de transporte da alternativa; TA - tempo médio de transporte da alternativa; VTA - confiabilidade da alternativa; CPA - custo médio com perdas de carga na operação da alternativa; $C E$ - consumo médio de energia da alternativa; EP - emissão média do poluente atmosférico pela alternativa; $\mathrm{ECO}$ - emissão média de $\mathrm{CO}_{2}$ pela alternativa.

As Figuras 3 e 4 apresentam os resultados da comparação das medidas de desempenho para cada uma das alternativas de forma isolada (por meio da aplicação da Equação 12) e agregada (por meio da aplicação da Equação 13), respectivamente. Em todos os casos, a alternativa de transporte especializado A1TE (rodo-ferro) é considerada como referência adotando o valor de 1,00 e quanto maior o valor obtido para cada uma das medidas de desempenho avaliadas para as demais alternativas, melhor seu desempenho, sendo candidata a ser considerada em substituição ao transporte especializado. 


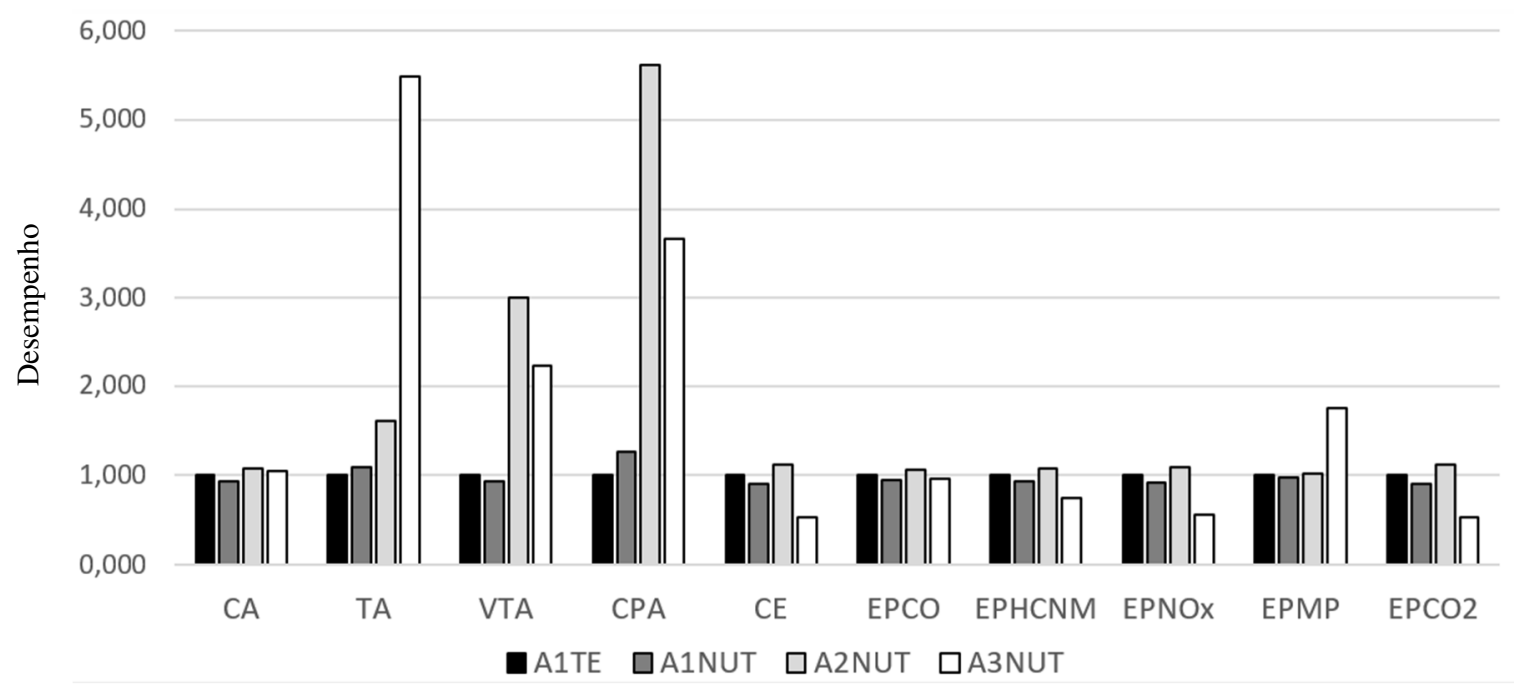

Figura 3. Resultado da comparação das alternativas de forma isolada

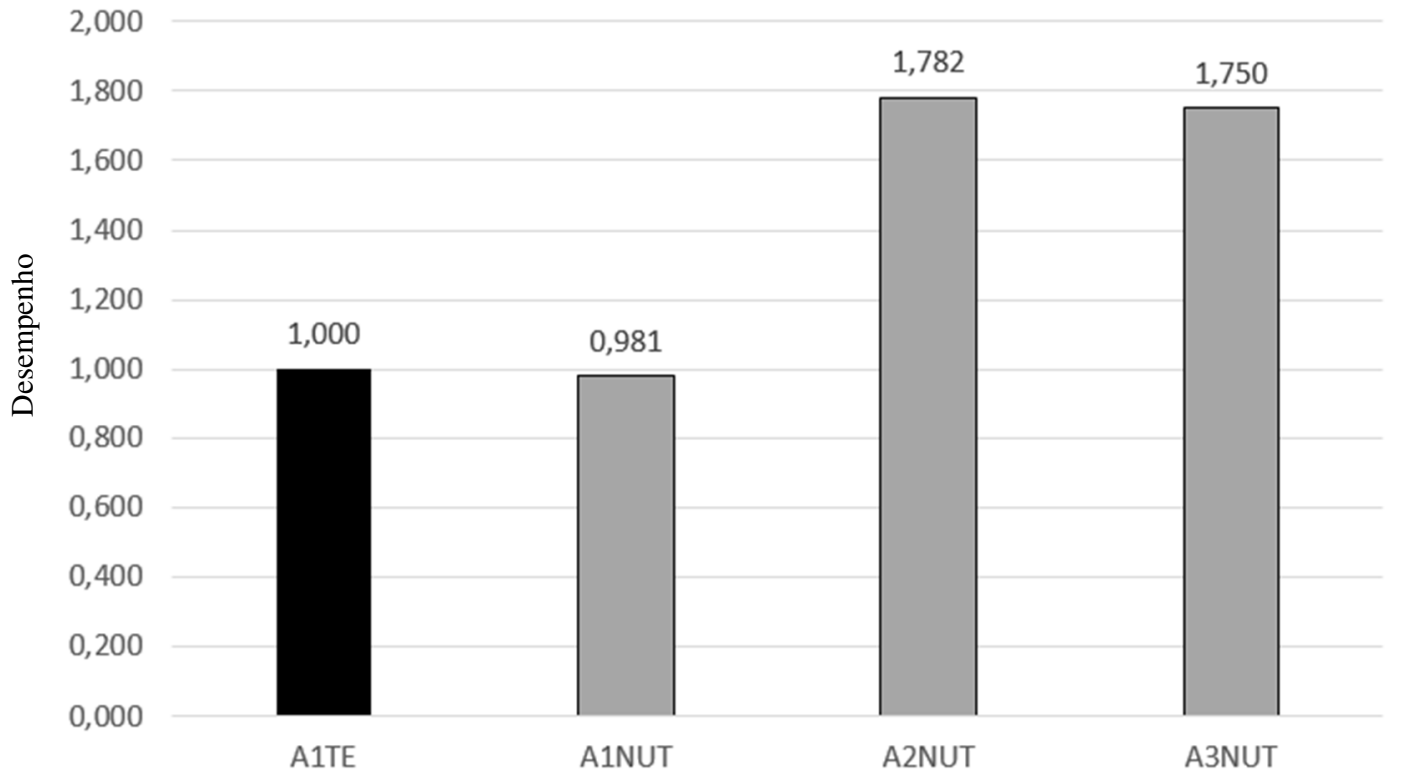

Figura 4. Resultado da comparação das alternativas de forma agregada

Da Figura 3 observa-se que A1nut apresenta resultados para as medidas de desempenho muito semelhantes aos obtidos para $\mathrm{A}_{1 \mathrm{TE}}$, com desempenho inferior em até 9\%, com excessão dos resultados obtidos para o custo com perdas de carga na operação, onde apresentou desempenho superior em $27 \%$ e tempo médio de transporte, com desempenho superior em 9\%. Este melhor resultado está associado ao fato desta alternativa realizar uma operação de transbordo a menos e metade da distância de transporte por esteira transportadora que A1TE conforme pode ser verificado na Tabela 2. Em função da introdução de $26 \mathrm{~km}$ adicionais $(+3,5 \%)$ de transporte rodoviário, os resultados obtidos para as medidas de desempenho de custos, confiabilidade, consumo de energia e emissão de poluentes atmosféricos e GEE's apresentaram valores piores se comparados aqueles obtidas para A1тE. Dessa forma, A1nut não atende ao principal requisito de ter desempenho igual ou superior a alternativa de transporte especializado para as medidas de desempenho associadas ao aspecto econômico (custo e nível de serviço). 
A alternativa que considera a combinação intermodal rodo-ferro-rodo e utiliza contêineres (A2NUT) apresenta desempenho superior para todas as medidas de desempenho, se comparada a A1тE. A utilização do maior segmento por ferrovia $(670 \mathrm{~km})$, do menor por rodovia $(26 \mathrm{~km})$, apresentar distância total de transporte $2 \%$ menor, a metade dos transbordos e o manuseio do produto ser realizado dentro de contêineres fechados, que minimizam as perdas por degradação do calcário, principal diferencial desta alternativa em relação às demais, leva a redução do custo em $8 \%$, do consumo de energia, oda emissão de $\mathrm{CO}_{2}$ em $12 \%$ e da emissão de poluentes atmosféricos entre 2\% (MP) e 9,5\% (NOx). Esta configuração privilegia em particular as três medidas de desempenho de nível de serviço (tempo médio de transporte, confiabilidade média e custo médio com perdas de carga na operação) que apresentam valores $60 \%, 200 \%$ e $460 \%$ superiores aos obtidos por $\mathrm{A}_{1 \mathrm{TE}}$, respectivamente.

0 transporte de calcário pelo modo rodoviário (A3NUT) apresenta desempenho superior a A1TE para todas as medidas de desempenho relacionadas ao aspecto econômico, em particular para aquelas associadas o nível de serviço, como tempo médio de transporte 4,5 vezes melhor que o obtido para $A_{1 \mathrm{TE}}$, reflexo de considerar uma distância $17 \%$ menor e realizar três transbordos a menos que A1TE (Tabela 2). Porém, o uso intensivo do transporte rodoviário, por distância 11,2 vezes maior que A1те, penaliza as medidas de desempenho relacionadas ao aspecto ambiental, apresentando consumo de energia, emissão de $\mathrm{CO}_{2}$ e de poluentes atmosféricos quase $89 \%$ maiores que as da alternativa de referência, com exceção para a emissão de material particulado (-80\%), reflexo da predominância do uso no Brasil de tecnologia de propulsão mais moderna em caminhões que em locomotivas.

Em uma avaliação agregada (Figura 4), onde a consideração dos aspectos econômico e ambiental é feita de forma equitativa, verifica-se que A1nut apresenta desempenho só 1,9\% inferior que $\mathrm{A}_{1 \mathrm{TE}}$, sendo equivalente a esta alternativa. Por outro lado, A2nut e A3nut, são, respectivamente, $78,2 \%$ e $75 \%$ melhores que $A_{1 \mathrm{TE}}$, fato que pode induzir a uma escolha equivocada, pois, como se observa por meio da Figura 3, A3NuT apresenta desempenho inferior para a maior parte das medidas de desempenho associadas ao aspecto ambiental que tradicionalmente não são consideradas pelos tomadores de decisão quando da escolha de alternativas de transporte.

Neste sentido é util avaliar a sensibilidade dos resultados obtidos quanto a consideração do aspecto ambiental na avaliação de desempenho das alternativas não usuais para o transporte de produtos de baixo valor agregado. A Figura 5 apresenta o resultado obtido quando se aplica uma escala de pesos $(100 \%$ a $0 \%)$ que vai de A100, situação hipotética quando só se consideraria o aspecto ambiental ( $100 \%$ de peso no aspecto ambiental e $0 \%$ de peso no aspecto econômico - sendo equivalente a só considerar na avaliação de desempenho as medidas associadas ao aspecto ambiental) na escolha das alternativas até E100 ( $0 \%$ de peso no aspecto ambiental e $100 \%$ de peso no aspecto econômico), situação usual, quando só se considera o aspecto econômico (custo e nível de serviço) para a escolha das alternativas. Os valores intermediários entre A100 e E100 (A90 a A10) são obtidos adotando X\% de peso para o aspecto ambiental e $100 \%$-X\% de peso para o aspecto econômico, com decrementos de $10 \%$ (p. ex: em A90 se aplica 90\% de peso para o aspecto ambietal e 10\% para o aspecto econômico).

Considerando A1Te como referência, assumindo valor igual a 1,00 ao logo de toda a escala de variação de pesos (A100 a E100), a partir da Figura 5 observa-se que A1nut é muito pouco sensível a indrodução progressiva dos aspectos ambientais em sua avaliação de desempenho, 
apresentando resultados muito próximos aos de A1TE para todas as situações consideradas (de A100 a E100), com variação relativa de $-12 \%$.

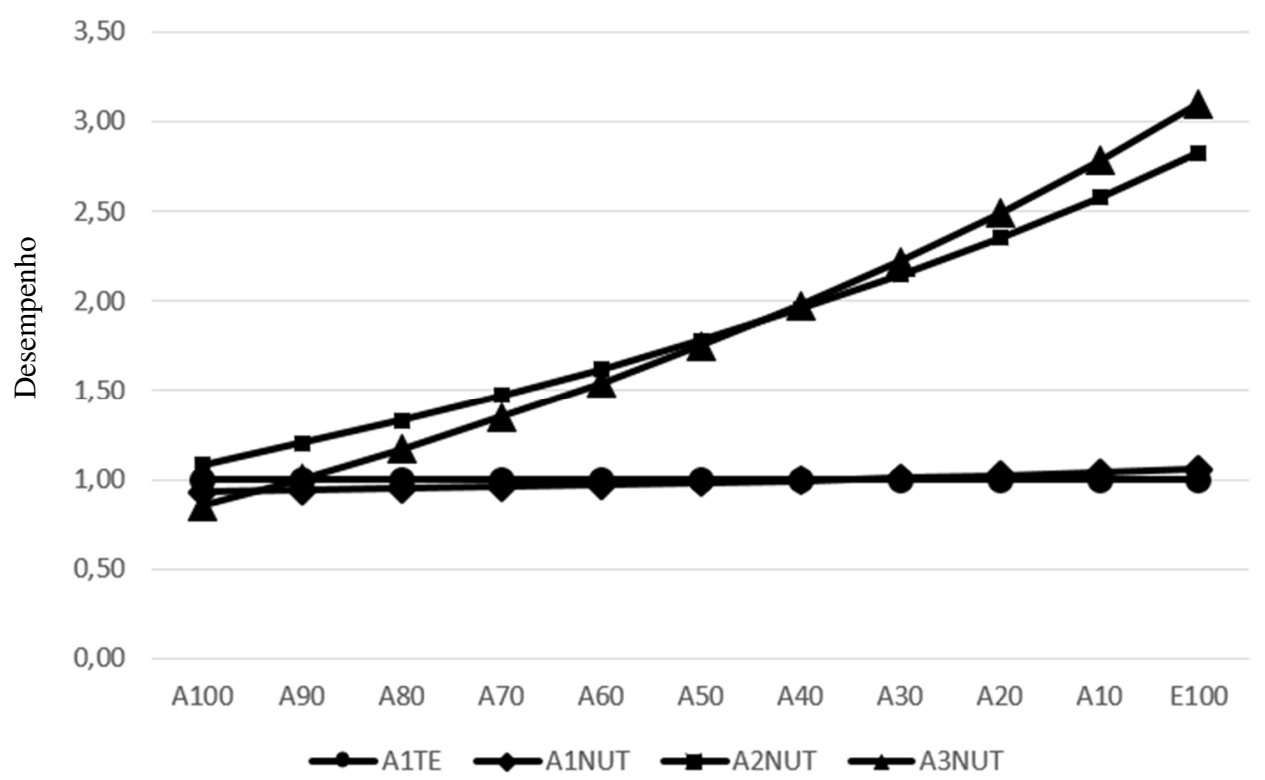

Legenda: $\mathrm{A}_{1 \mathrm{TE}}$ - alternativa de transporte especializado do tipo rodo-ferro; $\mathrm{A}_{1 \mathrm{NUT}}$ - alternativa de transporte não usual do tipo rodo-ferro-rodo; $\mathrm{A}_{2 \mathrm{NUT}}$ - alternativa de transporte não usual do tipo rodo-ferro-rodo com container e A3NUT - alternativa de transporte não usual do tipo rodoviário.

Fonte: elaboração própria.

Figura 5. Resultado da aplicação dos pesos para os aspetos ambiental e econômico

O comportamento observado para A1nut não ocorre para A2nUt e A3NUT, que apresentam, respectivamente, variação relativa de $-62 \%$ e $-73 \%$ se comparados os resultados de E100 com A100. Porém, o mais importante é que A2nut é a única alternativa que mantem desempenho superior a $A_{1 T E}(+8 \%)$ na situação A100. Observa-se da Figura 5 que embora A3nut apresente desempenho 9\% superior a A2NUT na situação E100, A3NuT é mais sensível à introdução do aspecto ambiental na sua avaliação, de forma A2nut torna-se melhor que A3NuT a partir da situação A40 (40\% de peso no aspecto ambiental e $60 \%$ de peso no aspecto econõmico), chegando a ficar $21 \%$ melhor que A3NuT na situação A100.

\section{CONSIDERAÇÕES FINAIS}

Os resultados apresentados a partir da aplicação do procedimento proposto demonstram ser possível escolher uma alternativa não usual para o transporte de produto de baixo valor agregado que apresente desempenho superior à alternativa de transporte especializado sob a ótica do aspecto econômico, considerando os indicadores de custo e nível de serviço, sem comprometer o desempenho do aspecto ambiental, situação imprescindível à garantia dos padrões atuais de responsabilidade ambiental das empresas. Com isso, entende-se que o objetivo proposto para este artigo tenha sido atingido.

O estudo mostra que A1TE é a alternativa especializada para o transporte de calcário no caso apresentado, porém para fazer a avaliação proposta foi necessário optar por três alternativas não usuais (A1NUT, A2NUT e A3NUT).

$\mathrm{Na}$ avaliação isalada, a A1NUT apresentou resultados muito semelhantes aos obtidos para A1TE. No caso da A2NUT, esta apresentou desempenho superior para todas as medidas de desempenho, se comparada a A1TE. A A3NUT apresentou desempenho superior a A1TE para 
todas as medidas de desempenho relacionadas ao aspecto econômico, porém quanto ao aspecto ambiental não se mostrou uma boa alternativa.

Na avaliação agregada, onde a consideração dos aspectos econômico e ambiental foi feita de forma equitativa, verificou-se que A1NUT também apresentou resultados muito semelhantes aos obtidos para A1TE. Por outro lado, A2NUT e A3NUT, se mostraram alternativas muito melhores, fato que poderia induzir a uma escolha equivocada, pois a A3NUT apresentou desempenho inferior para a maior parte das medidas de desempenho associadas ao aspecto ambiental.

Neste sentido optou-se por avaliar a sensibilidade dos resultados obtidos quanto a consideração do aspecto ambiental na avaliação de desempenho das alternativas não usuais. Aplicou-se uma escala de pesos (100\% a 0\%) que considerou A100, situação hipotética quando só se consideraria o aspecto ambiental e E100 situação hipotética quando só se consideraria o aspecto econômico.

Dessa forma, A2NUT foi a única alternativa que manteve desempenho superior a A1TE na situação A100. Embora a A3NUT tenha apresentado desempenho superior a A2NUT na situação E100, A3NUT se mostrou mais sensível à introdução do aspecto ambiental na sua avaliação, dessa forma a A2NUT tornou-se melhor que A3NUT a partir da situação A40, chegando a ficar melhor que A3NUT na situação A100.

O procedimento proposto é simples e de fácil aplicação, dependendo fundamentalmente da disponibilidade de dados obtidos a partir do monitoramento rotineiro das operações de transporte e da estimativa de fatores de emissão de poluentes atmosféricos e GEE que podem ser obtidos em inventários de emissões produzidos por instituições públicas. Estas características fazem com que sua aplicação seja relativamente barata e introduza pouco trabalho adicional ao que já é praticado par manter a operação dos sistemas de transporte. Excepcionalmente, para uma melhor acurácia dos resultados, os fatores de emissão podem ser medidos em campo, o que acarretará um trabalho de maior complexidade, custo e tempo.

Embora a aplicação do procedimento tenha considerado quatro alternativas de transporte, isto não representa uma limitação e um número maior de alternativas pode ser considerado, dependendo apenas da disponibilidade de dados e do aprimoramento do processo de cálculo. Para facilitar a aplicação do procedimento, recomenda-se que um banco de dados com valores representativos da operação de cada uma das alternativas de transporte seja mantido atualizado.

A consideração de outros aspectos ambientais, como a geração de ruídos e de resíduos líquidos e sólidos, bem como a introdução do aspecto social parece ser o aprimoramento natural a ser introduzido no procedimento de avaliação de desempenho de alternativas de transporte em trabalhos futuros.

\section{REFERÊNCIAS}

Almeida R.M. Análise empírica dos fatores determinantes e das estratégias de internacionalização da siderurgia brasileira. Dissertação de M.Sc., UFMG, Administração, Belo Horizonte, 2004.

ANTT (2016). Corredores Logísticos Multimodais Desenvolvimento de Redes Transeuropeias de Transporte e Implantação do Conceito do Corredor no Brasil. São Paulo, 2016.

Bektas, T., Ehmke, J. F., Psaraftis, H. N., \& Puchinger, J. (2018). The Role of Operational Research in Green Freight Transportation. European Journal of Operational Research.

Carvalho, C.R.V., Conceição Neto, A.S., Almeida, H.M., Fialho, L.B., Silva, M.V. Otimização da logística de abastecimento em uma indústria siderúrgica: um estudo de caso. Em: XXIV Encontro Nacional de Engenharia de Produção, Florianopolis, SC, Brasil, 2004. 
Castro. N. Estrutura, desempenho e perspectiva do transporte ferroviário de carga. Pesquisa e planejamento econômico, ppe, v.32, n.2, ago.2002.

Cazeri, G., Anholon, R., Ordoñez, R. E., \& Novaski, O. (2017). PERFORMANCE MEASUREMENT OF GREEN SUPPLY CHAIN MANAGEMENT: A LITERATURE REVIEW AND GAPS FOR FURTHER RESEARCH. Brazilian Journal of Operations \& Production Management, 14(1), 60-72. https://doi.org/https://doi.org/10.14488/BJOPM.2017.v14.n1.a7

CNT, 2011. Confederação Nacional do Transporte. Informe econômico do setor de transportes, 2011. Disponível em:<http://www.cnt.org.br/Imagens\%20CNT/PDFs\%20CNT/Informe\%20Econ\%C3\%B4mico/InformeEconomico072011.pdf> Acesso em 03 de dezembro de 2011.

Coelho Neto, G.; Ribeiro, P.C.C.. “Transporte em uma empresa da indústria siderúrgica brasileira”. In: XXVI ENEGEP, Fortaleza, CE, Brasil, 9-11 outubro, 2006.

D’Agosto, M. de A e Ribeiro, S. K. (2004). Eco-efficiency management program (EEMP) - a model for road fleet operation. Transportation Research D, 9, 497-511.

Demir, E., Bektaş, T., \& Laporte, G. (2014). A review of recent research on green road freight transportation. European Journal of Operational Research, 237(3), 775-793.

Feo-Valero, M.; García-Menéndez, L.; Sáez-Carramolino, L.; Furió-Pruñonosa, S. (2011) “The importance of the inland leg of containerised maritime shipments: An analysis of modal choice determinants in Spain"

Fontana, A., Faria, P., Moreira, M. E. P. e Montenegro, N. G. S. D. "Escolha do modal com maior índice socioeconômico ambiental para o transporte de soja a granel entre Cuiabá-MT e Santarém-PA”. In: XXII ANPET - Congresso de Pesquisa e Ensino em Transportes, Vitória, ES, Brasil, 2009.

Gonçalves, D. N. S.; D’Agosto, M. A. Cenários prospectivos futuros para o uso de energia em transportes no Brasil e as emissões de GEE Cenário Business as Usual (BAU) - 2050. 2017.

Guajardo, M. (2018). Environmental benefits of collaboration and allocation of emissions in road freight transportation. In Sustainable Freight Transport (pp. 79-98). Springer, Cham.

Holmgren, J.; Nikopoulou, Z.; Ramstedt, L.; Woxenius, J. (2014) “Modelling modal choice effects of regulation on low-sulphur marine fuels in Northern Europe".

IAB, 2011. Anuário Estatístico 2011. Rio de Janeiro: Instituto Aço Brasil, 2011.

Kerkkänen, A. Determining semi-finished products to be stocked when changing the MTS-MTO policy: case of a steel mill. Internatinal Journal Production Economics, pp. 111-118, August 2007.doi:10.1016/j.ijpe.2006.12.006

Leal Junior, I.C.; D`Agosto, M.A. "Modal choise evaluation of transport alternatives for exporting bio-ethanol from Brazil”. Transportation research part D, v. 16, pp.201-207, 2011b.

Leal Junior, I.C.; D`Agosto, M.A. "Modal choise for transportation of hazardous materials: the of land models of transport of bio-ethanol in Brazil". Journal of Cleaner Production. V. 19, pp. 229-240, Jan-Fev. 2011a.

Leitner, J. The political economy of raw materials transport from internal periphery to core in the early 20th century US. Journal of world systems research, V. 10, n. 2, pp 397-435, September 2004.

Lewis, P.E.; Rasdorf, W.; Frey, H.; Pang, S.; Kim, K.. "Requirements and incentives for reducing construction vehicle emissions and comparison of nonroad diesel engine emissions data sources". Journal of construction engineering and management. Vol. 5; pp. 341-351, 2009.

Marcucci, Edoardo. Regulating Transport in Europe. 2014.

MMA, 2011. 1o Inventário Nacional de Emissões Atmosféricas por Veículos Automotores Rodoviários, Ministério do Meio Ambiente, Brasília, DF.

Mohria S. S.; Haghshenasb H.; Salehic V. (2019). Two Levels Modelling of Container Choosing and Mode Choice between Road and Rail for Increasing the Containerized Rail Modal Share. Transportation Research Procedia, WORLD CONFERENCE ON TRANSPORT RESEARCH World Conference on Transport Research - WCTR 2019 Mumbai 26-31 May 2019.

Nealer, R.; Matthews, H. S.; Mellon, C. H.C. (2012) “Assessing the energy and greenhouse gas emissions mitigation effectiveness of potential US modal freight policies"

Panagakos, G.P.; Stamatopoulou, E. V.; Psaraftis, H. N. (2014) "The possible designation of the Mediterranean Sea as a SECA:A case study".

Pereira Jr, A.O. Matriz energética do estado do Rio de Janeiro: 2017-2031. Rio de Janeiro: Synergia, 2018.

Poso, A.T.. O processo de reestruturação da siderurgia mundial e brasileira: o caso da Companhia Siderúrgica Nacional. Dissertação de M.Sc., USP, São Paulo, SP, Brasil, 2007.

Ravibabu, M. (2013) “A nested logit model of mode choice for inland movement of export shipments: A case study of containerised export cargo from India"

Santos, G.A.G. Escolha de Alternativas para o Transporte de Calcário Siderúrgico Sob o Enfoque Ambiental. Dissertação de M.Sc.,COPPE/UFRJ, Rio de Janeiro, RJ, Brasil, 2012.

Vaz, S.L. A siderurgia brasileira a carvão vegetal: um estudo de arranjos verticais. Dissertação de M.Sc., USP, São Paulo, SP, Brasil, 2010.

Vernimmen, B.; Dullaert, W.; Willemé, P.; Witlox, F. (2008) “Using the inventory-theoretic framework to determine cost-minimizing supply strategies in a stochastic setting"

Wadud, Z. (2016) "Diesel demand in the road freight sector in the UK: Estimates for different vehicle types"

Wei, Y.; Liao, H.; Fan, Y. (2007). "An empirical analysis of energy efficiency in China's iron and steel sector”. Energy. Vol. 32, Issue 12, Pag. 2262-2270, December 2007. 
Weigel, M.; Fischedick, M.; Marzinkowski, J.; Winzer, P. (2016) “Multicriteria analysis of primary steelmaking technologies”.

Wolff, M., Abreu, C., \& Caldas, M. A. (2019). Evaluation of road transport: a literature review. Brazilian Journal of Operations \& Production Management, 16(1), 96-103. https://doi.org/https://doi.org/10.14488/BJOPM.2019.v16.n1.a9

Worrell, E., Price, L., Martin, N.. Energy efficiency and carbon dioxide emissions reduction opportunities in the US iron and steel sector. Energy Journal, v. 26, pp. 513-536, June 2001.

Woxenius, J.; Bergqvist, R. (2011) “Comparing maritime containers and semi-trailers in the context of hinterland transport by rail".

Alessandri, A., A. Di Febbraro, A. Ferrara and E. Punta (1998) Optimal Control of Freeways via Speed Signalling and Ramp Metering. Control Engineering Practice, v. 6, n. 6, p. 771-780. DOI: 10.1016/S0967-0661(98)00083-5. 